\title{
Characterizing the Signature of Flame Flashback Precursor Through Recurrence Analysis
}

\author{
Loizos Christodoulou, ${ }^{1, \text { a) }}$ Lipika Kabiraj, ${ }^{2, \text { b) }}$ Aditya Saurabh, ${ }^{2}$ and Nader Karimi ${ }^{1}$ \\ 1) School of Engineering, University of Glasgow, Glasgow, UK \\ ${ }^{2)}$ Hermann Föttinger Institute, Technische Universität Berlin, Germany
}

(Dated: 2 January 2016)

In this paper it is shown that prior to flashback, small dynamical changes appear in the system. These changes appear as a drift in the recurrence plots and are found to be associated with a gradual increase in the determinism and recurrence rate. Thus, this study indicates that precursors to flame flashback exist and can be detected in the multidimensional phase space reconstructed from pressure measurements acquired during flashback. This observation could have broad academic as well as industrial implications.

PACS numbers: 05.45.Tp, 47.70.Pq

Flame flashback is a complex phenomenon resulting from interactions involving multiple physical processes, each governed by its own inherent temporal and spatial scale. In addition, flashback is also a transient event, with the actual event lasting only fractions of a second. Investigation of flashback requires multiple, simultaneous diagnostics without prior knowledge of the relevant time and length scales of the physical processes involved. Detection, accordingly, deals with postevent characterization. In this paper, an attempt to detect subtle dynamics prior to flashbackwhich linear, time and frequency-domain methods cannot reveal-is made through recurrence analysis of the multidimensional phase space reconstruction from pressure time traces. Recurrence analysis is superior to the common techniques (e.g. spectra) used for the analysis of short, noisy datasets for the purpose of identifying dynamical changes. Following conventional recurrence analysis methods, different sections of pressure time traces from reacting flow conditions are analyzed. Recurrence plots and recurrence quantification measures immediately before flashback are compared to those long before flashback during the normal operation of the combustor. A comparison between recurrence characteristics at isothermal flow conditions and at reacting flow conditions is included to a) highlight the dynamics of the thermoacoustically unstable condition at which flashback occurs and b) to establish the limits of fluctuations in recurrence quantification analysis measures. It is observed that small drifts exist in the dynamical behavior of the system before flashback and are made apparent by recurrence methods. It is found that the changes span 2-4 seconds prior to flashback. Within this time

\footnotetext{
a) Electronic address: 1.christodoulou.1@research.gla.ac.uk

b) Electronic address: lipika.kabiraj@tu-berlin.de
}

frame, determinism and recurrence rate increase gradually as flashback is approached.

\section{INTRODUCTION}

Flame flashback is a concerning issue for the emerging lean premixed combustion technology that promises enhanced system efficiency and significant reduction in pollutant emission. Not only has the phenomenon been impossible to detect in advance, the complexity and the highly transient nature of the phenomenon make investigations intractable. Thus, understanding and suppression of this intricate issue has been a long standing issue and continues to challenge the research community as well as the industry.

Flashback refers to the unforeseen and instantaneous propagation of the reactive front to the upstream stages of the combustor ${ }^{1-3}$. Because upstream stages are not designed for the extreme conditions created by the flame, a flashback event could cause critical damages that must be avoided by system shutdown. Thus, the phenomenon is clearly undesirable and detecting the onset of flame flashback under various operating conditions, is an essential step in the design of premixed combustion systems.

The primary cause of flashback is the imbalance between the local flame and flow velocities. In a practical setting local flame and flow velocities depend on several physical processes that occur across different time and length scales. The physical processes involved include flame interaction with hydrodynamics (turbulence, boundary layer ${ }^{4,5}$ and coherent structures $\left.{ }^{6-8}\right)$, acoustics (thermoacoustic coupling) ${ }^{9-11}$, chemical kinetics ${ }^{2,12}$ and unsteady heat transfer. The referred works deal with the study of how the respective processes affect the flashback propensity of the system and/or the evolution of the individual processes as flashback takes place. It has been made clear that the physical mechanisms responsible for flame flashback are quite complex and are not yet sufficiently understood so as to efficiently eliminate the possibility of flashback. The transition from one dy- 
namical state, prior to flashback, to a totally different one corresponding to the state in which the flame is inside the premixing section can happen over an extremely short time (often a fraction of a second). Understanding the dynamics of such a transitional period is essential to the implementation of suppression mechanisms.

Due to the short temporal span of the transitional period, conventional time- and frequency-domain methods, become inaccurate in analyzing the system. Such techniques are inherently incapable of detecting the subtle complex features of the dynamics of flashback. Another problem associated with the highly complex and transient process is that without the prior knowledge of the temporal and spatial scales involved in flashback, in a given configuration, experimental diagnostics of the event (c.f. 13, 14) are difficult to perform. Changes prior to flashback are not apparent on direct visual or quantitative examination of time series and its spectral decomposition. Identification of the presence of system dynamics - and changes therein - that could be considered precursors to the event of flashback would, therefore, be of immense practical and academic interest.

The present work investigates the implementation of recurrence analysis of phase space trajectories reconstructed from pressure time traces for the detection of dynamical drifts and patterns that are expected to occur prior to, and could be related to flashback induced by thermoacoustic oscillations. Recurrence analysis, by definition, should be able to detect patterns of deterministic and non-deterministic dynamics that constitute changes in system dynamics that appear before a flashback event. It has been previously shown ${ }^{15,16}$ that phase space based methods, including recurrence analysis, can detect sudden changes associated with dynamical transitions in combustors. Likewise, with the help of recurrence analysis, it is shown here that prior to flashback, subtle dynamical changes occur in the system.

The article is organized as follows: The methods used to construct the recurrence plots and calculate the recurrence quantification analysis (RQA) measures, recurrence rate (RR), determinism (DET) and divergence (DIV) are described in section II. The experiment from which the existing pressure time series were recorded is described in section III. Subsequently, in section IV, the methods are applied to the existing pressure time series data from the experiment and the obtained results are discussed. In section V, a discussion summarizes the results and highlights their significance. Finally, concluding remarks, including the implications of the findings, are presented in section VI.

\section{METHODOLOGY}

Recurrence is a fundamental property of dynamical systems, discovered by Henri Poincaré ${ }^{17}$ in 1890 . Based on this property, Eckmann et al. ${ }^{18}$ introduced the concept of recurrence plots, which are graphical representa- tions of the recurrences of a systems' phase space trajectory. Visual information on system dynamics, contained in recurrence plots, can be quantified using recurrence quantification analysis. A recent comprehensive review of recurrence quantification analysis has been put forward by Marwan et al. ${ }^{19}$.

\section{A. Recurrence plot}

The recurrence plot for a time series $s(n)$, where $n=$ $1,2, \ldots, N_{t s}$ and $N_{t s}$ is the number of data points in the time series, is obtained in the following 4 steps.

1. Reconstruction of a phase portrait using time delay embedding. Each scalar measurement $s(n)$ is converted to a vector $\bar{s}(n)$ that specifies the position of the $n^{\text {th }}$ point in $D$ dimensional phase space with time delayed co-ordinates given by

$$
\bar{s}(n)=[s(n), s(n+T), \ldots, s(n+(D-1) T)]
$$

In the current work, the choice of the time delay, $T$, is made using average mutual information ${ }^{20}$ and the choice of the embedding dimension, $D$, is made through the method of false nearest neighbors ${ }^{20}$.

2. Selection of a recurrence threshold,

$$
0.03 d_{A} \leq \epsilon \leq 0.05 d_{A},
$$

where $\epsilon$ is the recurrence threshold and $d_{A}$ is the maximum attractor diameter. The threshold distance $\epsilon$ provides a criterion for trajectory recurrences. Two points in the phase space represent a recurrence if the distance between them which is calculated using an appropriate norm (in the present work using the $L^{2}$-norm) is less than or equal to the threshold distance $\epsilon$. A graphical representation is presented in Fig. 1.

Several approaches for the selection of an appropriate recurrence threshold have been suggested $^{19,21-24}$. The basic consideration for threshold selection is that the resulting RP is scarce while containing the essential features of the trajectory recurrences. A practical rule of thumb, which has been reported in literature ${ }^{21,25}$ is to fix the threshold close to $10 \%$ of the attractor radius. While this criterion has been found useful for a variety of dynamical systems, additional factors such as noise require a more involved study for the determination of an appropriate threshold ${ }^{19}$. For the system investigated here, the simple rule of thumb was found to be sufficient for the purpose of demonstrating that precursors to flashback exist. In the current work, we chose a recurrence threshold in the range $4-5 \%$ of the maximum attractor diameter (i.e. 8$10 \%$ of the corresponding attractor radius). 
3. Construct the recurrence matrix,

$$
R_{i, j}(\epsilon)=\Theta(\epsilon-\|\bar{s}(i)-\bar{s}(j)\|)
$$

where $\epsilon$ is the recurrence threshold, $\Theta(\arg )$ is the Heaviside step function: $\Theta(\mathrm{arg})=0$ if $\arg <0$ and $\Theta(\arg )=1$ if $\arg \geq 0,\|\arg \|$ is the $L^{2}$ norm and $i, j=1,2, \ldots, N_{p s}$ where $N_{p s}=N_{t s}-(D-1) T$ is the total number of points in the constructed phase space and $N_{t s}, D, T$ are the total number of data points in the time series, the embedding dimension and the time delay respectively.

To construct the recurrence matrix one starts from phase point $\bar{s}(i)$ with $i=1$ and calculates the distance $\|\bar{s}(1)-\bar{s}(j)\|$ between phase point $\bar{s}(1)$ and every other point in phase space $\bar{s}(j)$ where $j=1,2, \ldots, N_{p s}$. Every time the distance $\|\bar{s}(1)-\bar{s}(j)\|$ between the phase points $\bar{s}(1)$ and $\bar{s}(j)$ is calculated the following boolean operation is performed. If $\|\bar{s}(1)-\bar{s}(j)\| \leq \epsilon$, the points $\bar{s}(1)$ and $\bar{s}(j)$ define a recurrence and the entry $R_{1, j}=1$ is made in the recurrence matrix. Otherwise, the points do not define a recurrence and the entry $R_{1, j}=0$ is made in the recurrence matrix. This is equivalent to searching a neighborhood $\epsilon$ around phase point $i=1$ and checking which phase points $j=1,2, \ldots, N_{p s}$ fall into that neighborhood. Once the boolean operation has been completed for all $j$, one repeats the process for $i=2,3, \ldots, N_{p s}$. On completing this process for all $i$, one will have a $N_{p s} \times N_{p s}$ binary recurrence matrix $R_{i, j}$. It is noted that whether there is a recurrence at $(i, j)$ depends on the chosen recurrence threshold. To elucidate this the recurrence matrix is explicitly denoted in Eq.(3) as $R_{i, j}(\epsilon)$ and not simply as $R_{i, j}$.

The schematic in Fig. 1 shows examples of different entries made in a recurrence matrix for an arbitrary trajectory in phase space. Assume that a time series $s(n)$ with $n=1,2, \ldots, N_{t s}$ has resulted from an experimental measurement and by time delay embedding the data points $s(n)$ are converted to the time delayed coordinates $s(n), s(n+T), s(n+2 T)$. These define the positions of the points $i, j=$ $1,2, \ldots, 22$ in the phase space. For illustration, it has been assumed that $D=3$ and that $T$ and $N_{t s}$ have values such that the total number of points in the phase space is $N_{p s}=N_{t s}-(D-1) T=22$. The trajectory of the dynamical system that produced the time series is represented by the continuous line that connects the phase points. The shaded balls have a radius $\epsilon$ and are the neighborhoods of phase points $i, j=5,13,15,17$. In the neighborhood of phase point $i=5$ there are no phase points $j$ except for $j=5$ (the phase point itself). Thus, $R_{5, j}=0$

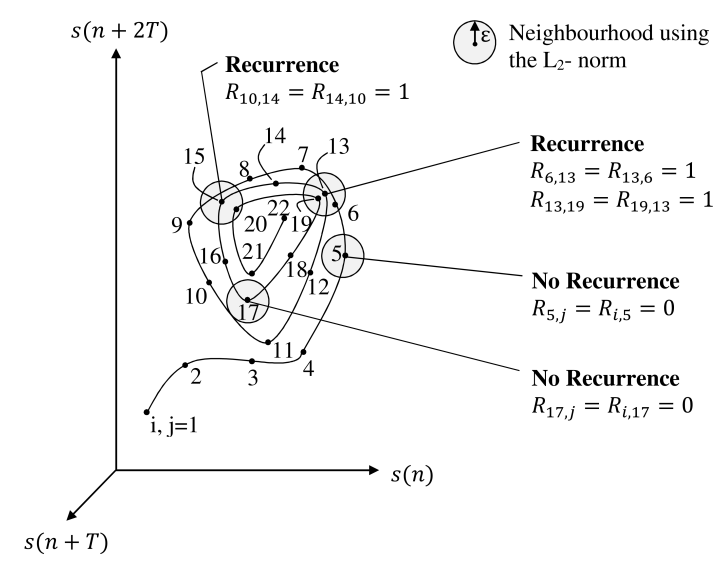

FIG. 1. Examples of entries in the recurrence matrix $R_{i, j}$ when $i \neq j$. for an arbitrary trajectory in phase space

for all $j$ except $j=5$. Because all the neighborhoods have the same radius $\epsilon$ when $j=5$ then $R_{i, 5}=0$ for all $i$ except $i=5$. For $i=j=5$ then $R_{5,5}=1$. In the neighborhood of the phase point $i=13$ there are two phase points $j=6$ and $j=19$. Thus, $R_{13,6}=1$ and $R_{13,19}=1$. Again, because all neighborhoods have the same radius $\epsilon$ the recurrence matrix is symmetric and therefore $R_{6,13}=1$ and $R_{19,13}=1$.

4. Plot the recurrence matrix, Eq. 3 using different colors for its binary entries, e.g., plotting a black dot at the coordinates $(i, j)$, if $R_{i, j}=1$ and a white dot, if $R_{i, j}=0$. Since $R_{i, j}=1$ for $i=j$ by definition, the RP has always a black main diagonal line, the line of identity (LOI). Furthermore, the $\mathrm{RP}$ is symmetric by definition with respect to the main diagonal (i.e. $R_{i, j}=R_{j, i}$ ) when a constant $\epsilon$ is used.

The appearance of a RP depends on the underlying dynamics of the system that produced the time series from which the RP is constructed. Small scale and large scale structures in the RP are related to specific dynamic behaviors ${ }^{19}$. For example diagonal lines in a RP are a sign of deterministic dynamics because they occur when the state space trajectory of the system returns to a previously visited state and then follows much the same path as it did before. The diagonal lines increase in length as long as the state space trajectory keeps following the previously followed path. Thus, the number of diagonal lines in the RP can be correlated to the degree of determinism exhibited by the dynamics of the system.

In the current study a series of MATLAB codes were developed to implement steps 1-4 on one second long, non-overlapping windows of the time series under investigation. 


\section{B. Recurrence quantification analysis}

Recurrence quantification analysis (RQA) introduces a statistical measure of DET, that exploits this observation in order to quantify the determinism of the system. The determinism, DET, is defined as,

$$
D E T=\frac{\sum_{l=l_{m i n}}^{N_{p s}} l P(l)}{\sum_{l=1}^{N_{p s}} l P(l)}
$$

where, $l$ is the length or number of black dots that form the diagonal lines, $l_{\text {min }}$ is a threshold diagonal line length, and $P(l)$ is the histogram of diagonal lines of length $l$. The DET measure is simply the ratio of the number of black dots in the RP that form diagonal lines to the total number of black dots in the RP. The threshold diagonal line length $l_{\text {min }}$ is defined to avoid counting false recurrences, such as those associated with diagonal lines that are a consequence of tangential motion. Tangential motion refers to the situation where a recurrence is recorded falsely as a consequence of the neighborhood $\epsilon$ encapsulating one or more phase points that are before or after the current phase point whose neighborhood is being searched and are on the same trajectory segment. Tangential motion can be the result of using a large $\epsilon$ i.e. searching a large area and/or using a time series that has been sampled at high frequency in which case the phase points in the reconstructed phase space are densely packed along the trajectory. In the present work $l_{\text {min }}=2$ is used for the calculation of $\mathrm{DET}^{26}$. In addition to DET the RQA measures RR and DIV are also calculated in the present work.

The recurrence rate $R R$ is defined as,

$$
R R=\frac{1}{N_{p s}^{2}} \sum_{i, j=1}^{N_{p s}} R_{i, j}
$$

where, $R_{i, j}$ is the recurrence matrix. The $\mathrm{RR}$ is the density of recurrence points in the RP. A more physical interpretation is that it is the probability of the dynamical system returning to a previous state.

The divergence DIV is defined as,

$$
D I V=\frac{1}{L_{\max }}
$$

where, $L_{\max }$ is the length of the longest diagonal line in the RP excluding diagonal lines of length $l<l_{\text {min }}$. Thus, DIV is the reciprocal of the length of the longest diagonal line in the RP. It indicates how fast the trajectories diverge in phase space. A small $L_{\max }$ is an indication that the trajectories in phase space diverge very fast and this results in DIV being large. On the other hand if $L_{\max }$ is large then the trajectories diverge slowly from each other and DIV is small.

The line of identity (LOI) is not included in the calculation of the RQA measures. The recurrence matrices obtained from the MATLAB code for each one second non-overlapping window of the pressure time series from the cold and hot flows have been used to calculate the RQA measures DET, RR and DIV using Eq. 4, Eq. 5 and Eq. 6 respectively.

\section{EXPERIMENT}

The present work uses the pressure time series obtained from a recent experiment on a laboratory scale burner. The burner has been used in studies in the past ${ }^{27,28}$. A schematic of the burner is shown in Fig. 2. The burner consists of a long circular tube connected at the upstream end to a plenum chamber (not shown in Fig. 2) and at the downstream end to a brass flame enclosure. A central rod runs through the entire length of the plenum and the tube and supports a conical bluff-body at the downstream end of the tube. The edge of the bluff-body is flush with the exit plane of the tube. Upstream of the bluff-body there is a swirler and further upstream there are layers of fine mesh which act as flame traps and, in the event of flashback, prevent deep penetration of the flame into the upstream sections.

During an experimental run, air and fuel (pure methane) streams are carefully measured through mass flow controllers and are premixed prior to entering the burner. A few layers of honeycombs upstream of the tube ensure the existence of a uniform and fully developed flow. The air and fuel mixture travels down the tube that contains the mounted pressure tapping before it passes through the swirler and reaches the conical bluffbody at the downstream end of the tube. Under normal operating conditions the flame is stabilized on the edge of the bluff-body and the flame is inside the flame enclosure. After the occurrence of flame flashback the flame is inside the tube at the location of the flame traps.

The time series used in the present work are pressure measurements conducted using a pressure trans-

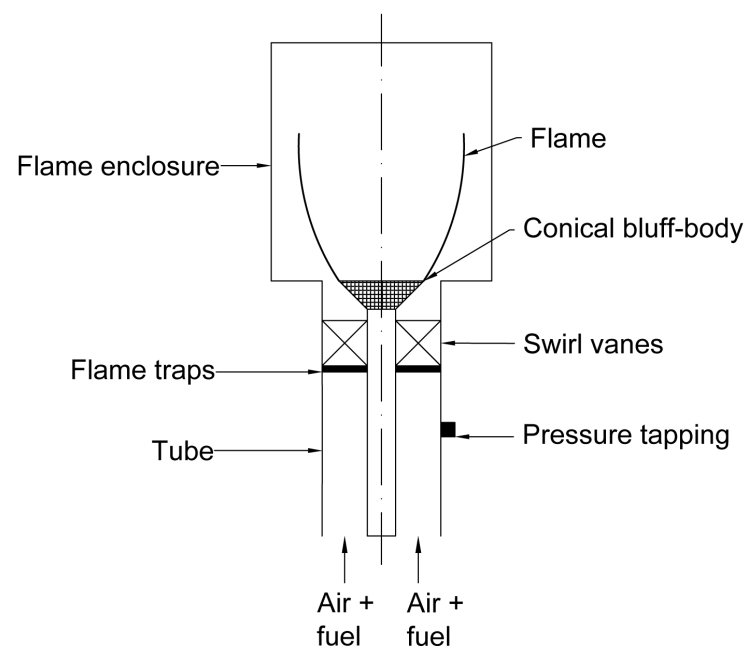

FIG. 2. Burner schematic. 
TABLE I. The operating conditions for the experiments and the notation used for the presented cases.

\begin{tabular}{cccc}
\hline \hline \multicolumn{2}{c}{ Pressure time series } & $\mathbf{U}$ & $\phi^{*}$ \\
No flame & With flame $^{*}$ & $\left(\mathbf{m s}^{-\mathbf{1}}\right)$ & $(-)$ \\
\hline$p_{10}$ & $P_{10}$ & 10 & 0.79 \\
$p_{12}$ & $P_{12}$ & 12 & 0.73 \\
$p_{14}$ & $P_{14}$ & 14 & 0.74 \\
\hline \hline
\end{tabular}

ducer (Kulite $\mathrm{XCS} 093^{1}$ ) positioned on the wall of the tube upstream of the swirler. Three pressure time series were recorded from the cold flow with velocities of $U=10 \mathrm{~ms}^{-1}, U=12 \mathrm{~ms}^{-1}, U=14 \mathrm{~ms}^{-1}$ (measured at the top edge of the bluff-body) and three pressure time series were recorded from the hot flows for the same velocities.

In the case of the hot flow the equivalence ratios were $\phi=0.79, \phi=0.73$ and $\phi=0.74$ for the three flow velocities $U=10 \mathrm{~ms}^{-1}, U=12 \mathrm{~ms}^{-1}$ and $U=14 \mathrm{~ms}^{-1}$ respectively. The pressure measurements were sampled at $8192 \mathrm{~Hz}$ and $16384 \mathrm{~Hz}$ from the cold and hot flows respectively.

The average flow properties were kept constant during all runs of the experiment in cold and hot flows. Amongst the components of the burner, which experience significant warming up, the flame enclosure features the highest thermal inertia and, therefore the longest thermal transient time. Continuous temperature monitoring of the flame enclosure, during the reactive tests, revealed that the thermal transient time of the enclosure was less than $60 \mathrm{~s}$. Hence, to ensure that the observed dynamics were not affected by the transient thermal effects, the pressure measurements were started at least $90 \mathrm{~s}$ after the ignition of the burner. Further, to eliminate the initial thermal effects in the flame enclosure, prior to the ignition of the flame, the burner was left to cool down to room temperature. For all investigated cases, the system was thermoacoustically unstable featuring large pressure oscillations. The thermoacoustically unstable state was followed by flame flashback and subsequent stabilization in the swirler. The fuel flow was cut shortly after flame flashback.

The pressure time series recorded during the experiment runs are summarized in Table I. The following notation convention is adopted for making reference to the time series. A pressure time series recorded from the cold flow with flow velocity $U$ is referred to as time series $p_{U}$. Similarly, a pressure time series recorded from the

\footnotetext{
1 Specifications: pressure range 0.35 bar, rated electrical excitation $10 \mathrm{VDC} / \mathrm{AC}$, input impedance $1000 \mathrm{ohm}$ (Nom), full scale output $200 \mathrm{mv}$, residual imbalance 5mv (Typ), combined nonlinearity, hysteresis and repeatability 0.1\% FSO (Typ), Natural frequency $150 \mathrm{kHz}$. See Ref. 29 for information regarding the frequency response characteristics of the employed pressure sensor.
}

hot flow with flow velocity $U$ is referred to as time series $P_{U}$. Therefore, lower case $\mathrm{p}$ for the cold flow, upper case $\mathrm{P}$ for the hot flow and the subscript denotes the velocity of the flow.

\section{RESULTS}

\section{A. Isothermal flow}

Pressure oscillations in the isothermal flow correspond to acoustics induced in the combustor by the turbulent flow. The pressure time series $p_{10}$ from the cold flow is shown in the top frame of Fig. 3. The amplitude of the pressure oscillations which can be characterized as broadband noise is about $10 P a$. The broadband peaks correspond to the acoustic resonance frequencies of the combustor. This is visible in the spectrogram of $p_{10}$ that is shown in the bottom frame of Fig. 3, with the dark bands appearing at acoustic resonance frequencies. The same observations were made for the pressure fluctuations in the higher flow velocity cases, $p_{12}$ and $p_{14}$, which are not shown here.

Correspondingly, the recurrence plot obtained from the cold flow time series, shown in Fig. 4, resembles salt and pepper noise: a homogeneous distribution of single isolated dots. A few short segments that can be observed are again a result of the acoustic resonance induced intermittently by the turbulent flow. This is in line with the spectrogram in Fig. 3. The cold flow features are in contrast with those of the reacting conditions presented next, and are presented to illustrate how thermoacoustic coupling in the presence of the flame induces deterministic behavior in the system.
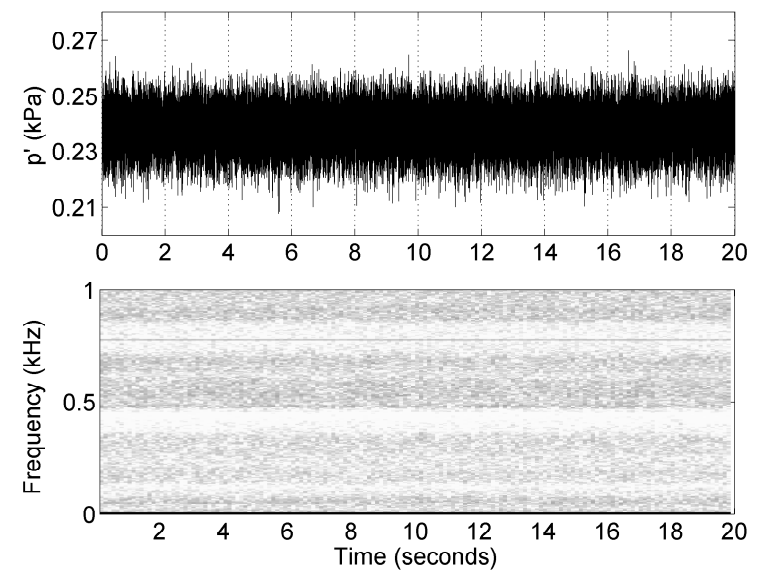

FIG. 3. The pressure time series $p_{10}$ from the cold flow and the corresponding spectrogram. The darker the color in the spectrogram, the higher the amplitude of the corresponding frequency component. In this case, the distribution is roughly uniform: broadband characteristics. 


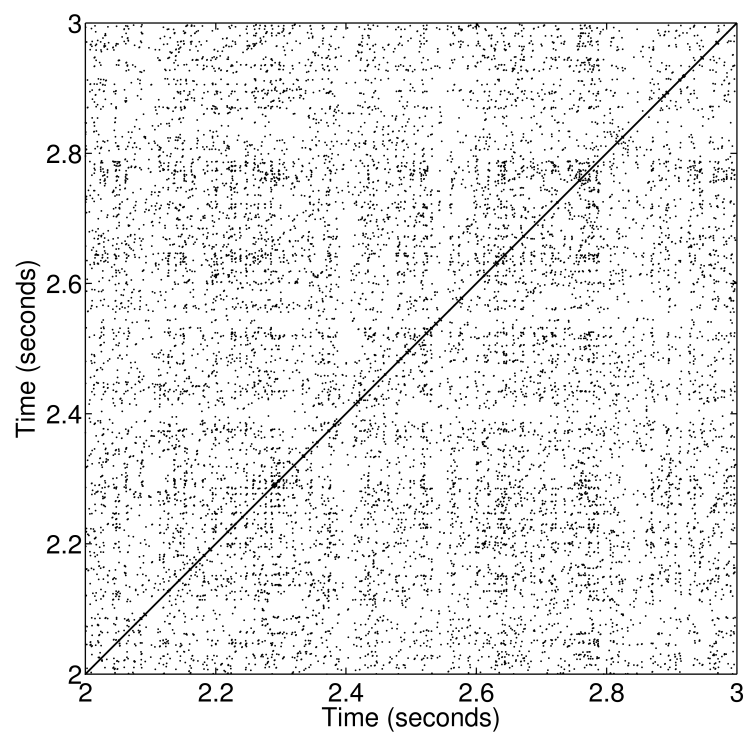

FIG. 4. The recurrence plot constructed for a one second window of the pressure time series $p_{10}$ from the cold flow using the parameters $T=4, D=5$ and $\epsilon=0.05 d_{A}$.

\section{B. Reacting flow: time and frequency analysis}

For the reacting conditions, the pressure time series $P_{10}, P_{12}$, and $P_{14}$ with their corresponding spectrograms are shown in Fig. 5(a)-(c), respectively. The amplitude of the pressure oscillations is about $1-2 k P a$ before flashback. The jump in the amplitude of the pressure oscillations seen in the time series in Fig. 5 corresponds to the flame flashback event. After flashback, the flame anchors in the upstream tube at the flame traps and the change results in high amplitude instability. The approximate time instant at which the amplitude of the pressure oscillations becomes large will be referred to hereafter as the approximate flashback point $t_{\text {flash }}$. The arrows in Fig. 5 indicate the approximate flashback point of each time series. It should be noted that the flashback event is an extremely fast process and it is, therefore, difficult to precisely determine the instant when it occurred in the pressure time trace. In the scale of the pressure traces in Fig. 5 the jump appears almost instantaneous and it is therefore convenient for the purpose of discussion to define the time instant of the jump as the nominal approximate flashback point. The approximate flashback points for the time series $P_{10}, P_{12}$, and $P_{14}$ are at $t_{\text {flash }}=6 s$, $t_{\text {flash }}=16 \mathrm{~s}$ and $t_{\text {flash }}=5 \mathrm{~s}$ respectively. Data acquisition was stopped shortly after the occurrence of the flashback.

The pressure time series, $P_{10}$, and the corresponding spectrogram in Fig. 5(a) indicate that prior to flame flashback, two dominant frequencies around $270 \mathrm{~Hz}$ and $390 \mathrm{~Hz}$ are present. The power levels of the oscillations are inferred by the darkness of the lines in the spectrogram (the darker the line in the spectrogram the higher is the power of the oscillations at the particular frequency).
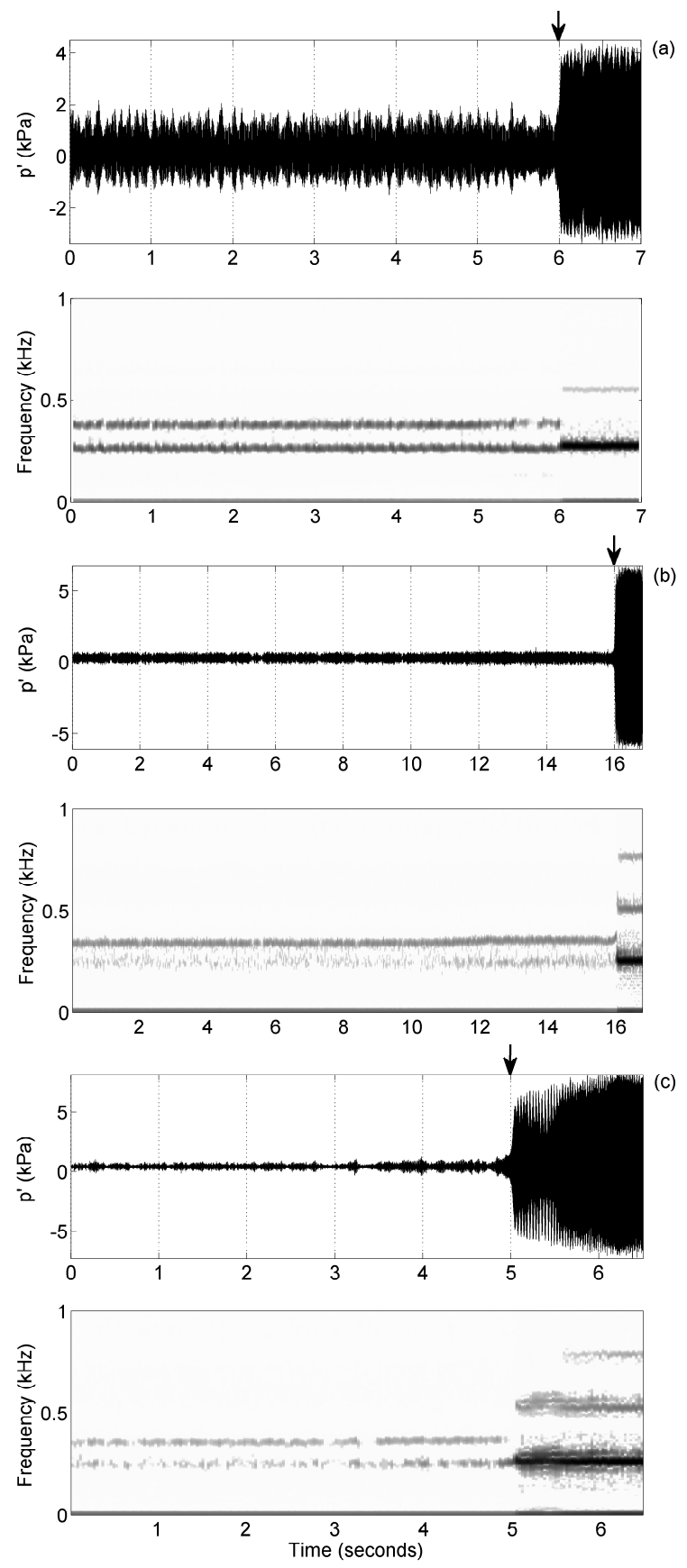

FIG. 5. The pressure time series (a) $P_{10}$, (b) $P_{12}$ and (c) $P_{14}$ from the hot flows. A spectrogram is shown below the corresponding time series. The vertical arrows on top of the pressure time series plots indicate the approximate instant at which flame flashback occurs.

The pressure oscillations prior to flashback at the two frequencies seem to be at approximately the same power level. After flame flashback, again the pressure oscillates at two dominant frequencies (around $270 \mathrm{~Hz}$ and $540 \mathrm{~Hz}$ ). The lower frequency after flashback $(270 \mathrm{~Hz})$ is the same as the lower frequency found prior to flashback, and the higher frequency $(540 \mathrm{~Hz})$ is the first harmonic. In the 
case of the $P_{12}$ time series in Fig. 5(b) there are two dominant frequencies prior to flashback (around $270 \mathrm{~Hz}$ and $340 \mathrm{~Hz}$ ) and after flashback there are three dominant frequencies $(270 \mathrm{~Hz}$ and the first and second harmonics). In the case of the $P_{14}$ time series in Fig. 5(c) there are two dominant frequencies prior to flashback $(270 \mathrm{~Hz}$ and 360 $\mathrm{Hz})$ and after flashback there are again three dominant frequencies ( $270 \mathrm{~Hz}$ and the first and second harmonics). An important point to note regarding the spectrograms is that near the time of flame flashback one can see a sudden change in the dominant frequencies. The spectrograms offer no indication that the point of flashback is being approached.

\section{Reacting flow: recurrence plots}

The recurrence plot for the one second window of the $P_{10}$ pressure time series from the reacting flow, two seconds prior to the approximate flashback point is shown in Fig. 6. The increased proportion of short line segments - deterministic structures - interrupted by isolated points or white horizontal and vertical spaces is clearly visible. Short line segments indicate a positive maximal Lyapunov exponent ${ }^{18,19}$, and hence, chaotic dynamics of the thermoacoustic coupling, that have also been identified in other studies ${ }^{15,28,30}$. In order to describe the distribution of line segments in the RP, a range of line lengths will be required. From the point of view of information theory, this means that a high level of complexity exists in the system dynamics. The vertical (or horizontal) white spaces indicate the presence of transients that occur roughly at intervals of 1-2 seconds. While the RP shown in Fig. 6 spans only one second of the time series, the RP for earlier sections of the pressure time trace are qualitatively similar. The observation also holds for $P_{12}$ and $P_{14}$.

The pattern formed by the collection of points and diagonally aligned line segments is a signature of the particular state under discussion $\left(P_{10}\right.$ long before the flashback event) and is different for the other cases, $P_{12}$ and $P_{14}$, primarily due to different flame dynamics and turbulence intensity of the base flow.

The recurrence plots obtained for the one second windows of the time series $P_{10}, P_{12}$, and $P_{14}$ immediately prior to flashback (the right hand edge of the one second window coincides with the approximate flashback point of the time series) are shown with the corresponding sections of the time series in Fig. 7(a)-(c) respectively. The parameters used to obtain the recurrence plots in Fig. 7 are summarized in Table II. The values of $T$ and $D$ were obtained by applying the methods mentioned in point 1 of section II A to the first $0.3 \mathrm{~s}$ of each pressure time series. The values of $T$ and $D$ were found to saturate when a sample space greater than the conservative amount of 4900 data points $(\sim 0.3 s$ given the sampling frequency of $16384 \mathrm{~Hz}$ ) was used. Furthermore, the values of $T$ and $D$ were found to be the same when computed for different

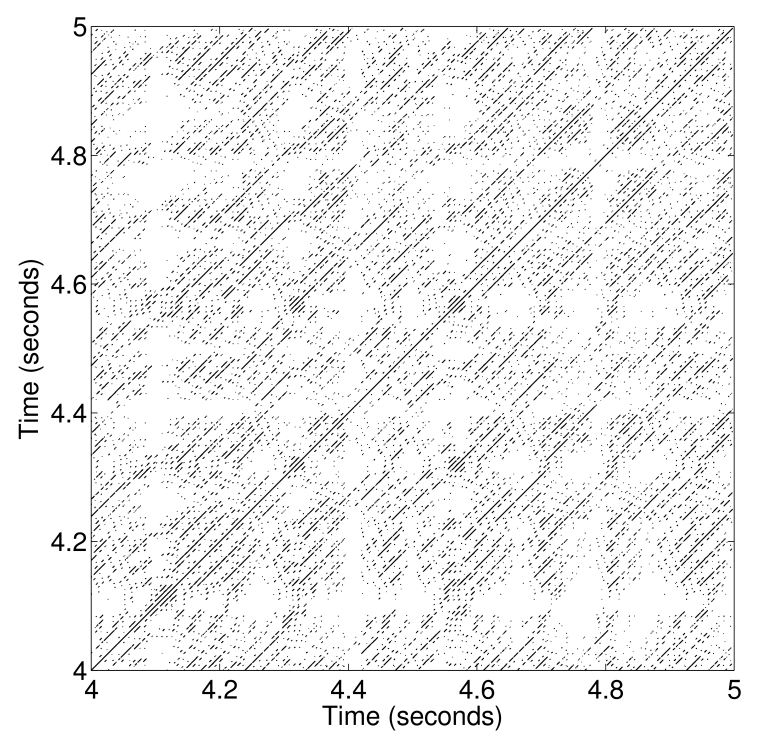

FIG. 6. The recurrence plot constructed for a one second window of the pressure time series $P_{10}$ from the hot flow using the parameters $T=15, D=4$ and $\epsilon=0.05 d_{A}$. The window of the time series is between $t=4 \mathrm{~s}$ and $\mathrm{t}=5 \mathrm{~s}$ and is some time prior to flashback.

TABLE II. The parameters used to obtain the recurrence plots shown in Fig. 7 and the RQA measures shown in Fig. 9.

\begin{tabular}{cccc}
\hline \hline Pressure time series & $\begin{array}{c}\text { T } \\
\text { (data points) }\end{array}$ & $\begin{array}{c}\mathbf{D} \\
(-)\end{array}$ & $\begin{array}{c}\epsilon \\
\left(\% \mathbf{d}_{\mathbf{A}}\right)\end{array}$ \\
\hline$P_{10}$ & 15 & 4 & 5 \\
$P_{12}$ & 14 & 6 & 4 \\
$P_{14}$ & 13 & 6 & 4 \\
\hline \hline
\end{tabular}

$0.3 s$ long sections of the time series as long as the sections were prior to flashback.

It is immediately apparent that the recurrence plot in Fig. 7(a), obtained for the $P_{10}$ pressure time series, contains more black dots that form diagonal lines than the other two recurrence plots obtained for the $P_{12}$ and $P_{14}$ pressure time series. This implies, that immediately prior to flashback, there is more determinism in the flow from which $P_{10}$ has been recorded. The reason for this is believed to be the less turbulence in the base flow in the case of the $P_{10}$ measurement as opposed to the base flows in the case of the $P_{12}$ and $P_{14}$.

\section{Flashback precursors in recurrence plots}

The pattern seen in the recurrence plot in Fig. 7(a) is for a one second long window of the $P_{10}$ time series just before the approximate flashback point. The pattern is notably different from that in recurrence plots developed for earlier windows of $P_{10}$ (see Fig. 6, for instance). Thus, the dynamics of the system are quite different just before 

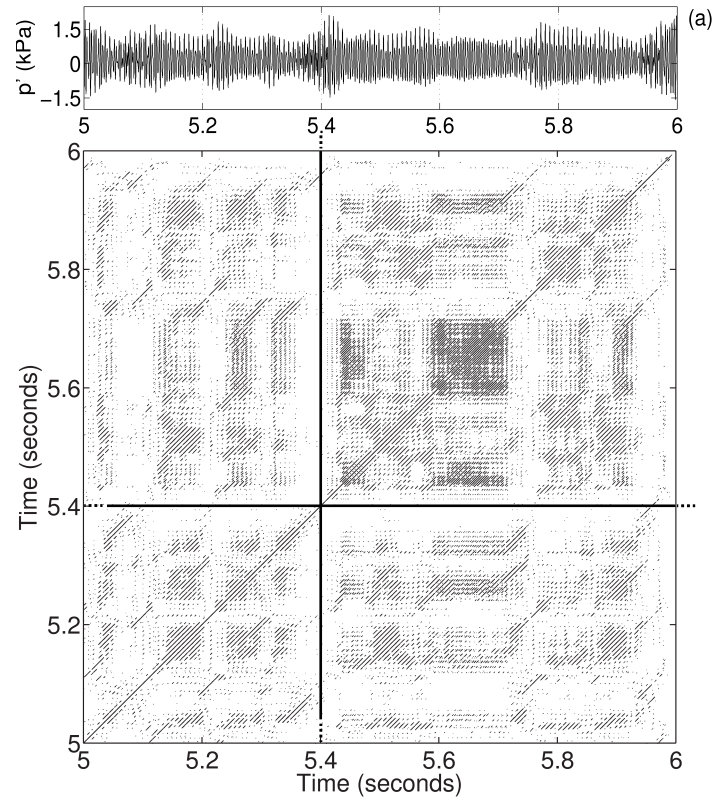
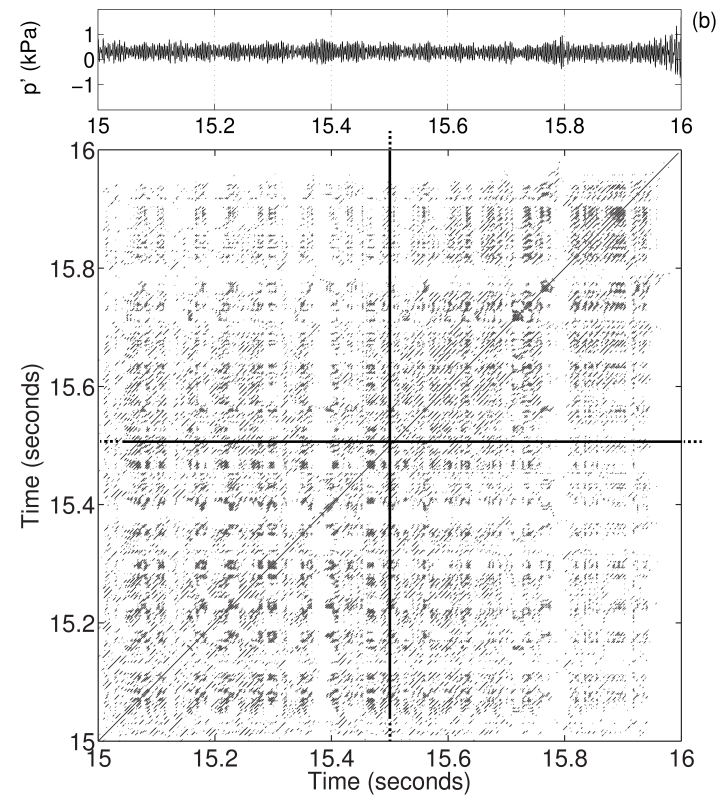

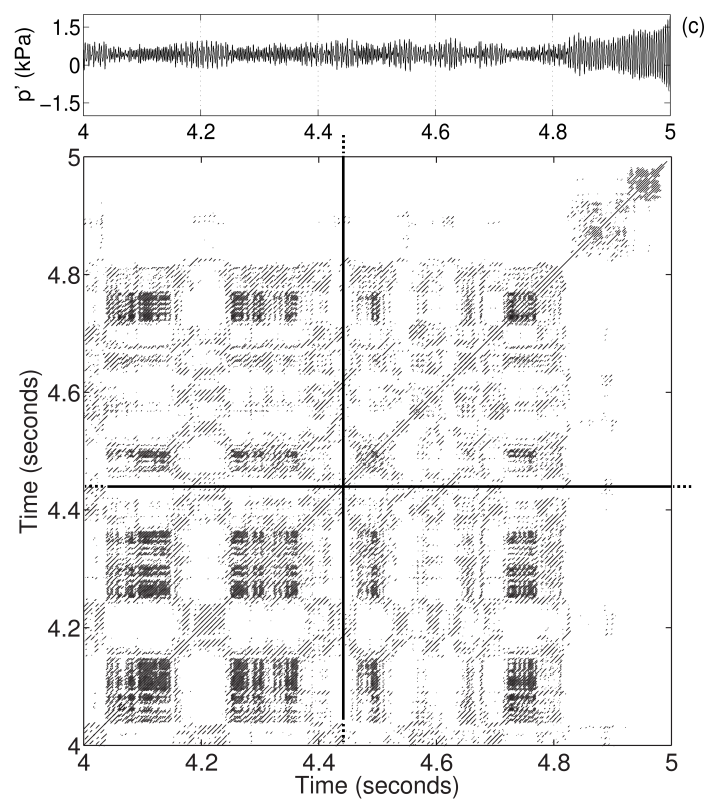

FIG. 7. Recurrence plots constructed for the one second window of the pressure time series (a) $P_{10}$, (b) $P_{12}$ and (c) $P_{14}$ immediately prior to flashback. The solid lines separate the RP along the main diagonal into two parts with visually differing texture.

flashback. The same is observed for the $P_{12}$ and $P_{14}$ pressure time series.

Further inspection of each individual recurrence plot in Fig. 7 reveals that there are changes of the pattern within the one second windows as one moves diagonally upwards and approaches the approximate flashback point (the right hand edge of each RP). To illustrate this, the RPs in Fig. 7 are divided into four sections by horizontal and vertical lines: the pattern seen in the lower left section of any RP Fig. 7 is different to the pattern seen in the upper right section. Towards the end of the one second windows, a small region of a transient can be identified (the horizontal and vertical white patches). This region is the largest for the case, $P_{14}$. This transient signature is due to the change in amplitude of the pressure time trace as a result of the flashback event. Thus, the flashback event can be clearly identified in the RP. More interestingly, the RP also contains smaller changes even earlier: for $P_{10}$, the solid vertical and horizontal rules at $\sim 5.42 \mathrm{~s}$ in Fig. 7(a) separate two regions along the main 


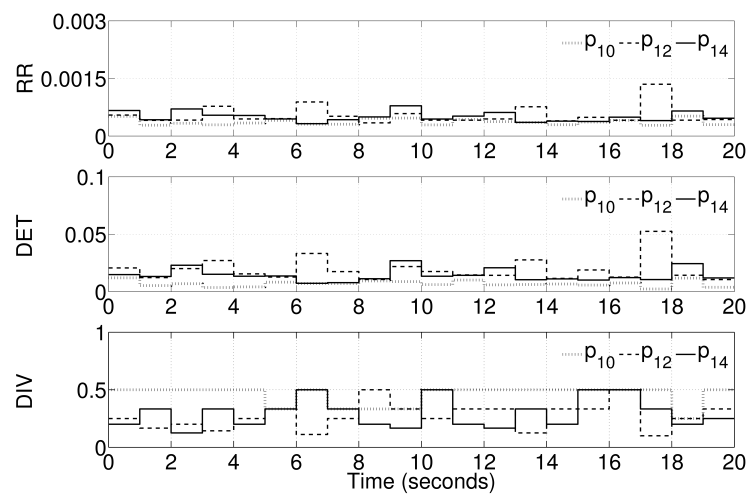

FIG. 8. The three RQA measures, recurrence rate (RR), determinism (DET) and divergence (DIV) calculated for the pressure time series $p_{10}, p_{12}$, and $p_{14}$. The RQA measures are calculated for one second, non-overlapping windows of the pressure time series.

diagonal that are visually distinguishable. The same can be inferred for the RPs of cases $P_{12}$ and $P_{14}$.

As we have seen, the structure of the RPs in Fig. 7 clearly changes as the point of flashback is approached (i.e. as one moves upwards along the diagonal of the RP). However, no clear sign that flashback is approaching is evident by inspection of the time series sections corresponding to the RPs (shown above each RP in Fig. 7). These changes observed in the structure of the recurrence plots immediately prior to flame flashback could be an indication that flashback is about to occur: flashback might have precursors associated with subtle dynamical changes in the system.

\section{E. Flashback precursors in recurrence quantification measures}

Further support to this observation is obtained from recurrence quantification analysis (RQA) ${ }^{18,19,31}$.

Plots showing the calculated RQA measures, the recurrence rate (RR), determinism (DET), and divergence (DIV) for the pressure time series $p_{10}, p_{12}$, and $p_{14}$ from the cold flow and $P_{10}, P_{12}$, and $P_{14}$ from the hot flow are shown in Figs. 8 and 9 respectively. The RQA measures are calculated using one second long non-overlapping windows of the pressure time series for the cold flow, and one second, $50 \%$ overlap windows for the hot case. Increasing the overlap would lead to further smoothing of the RQA curves. Further, the recurrence thresholds shown in Table II are fixed for the RQA calculations (i.e. the same recurrence threshold is used for all windows of each time series).

The RQA measures of the pressure time series $P_{10}, P_{12}$, and $P_{14}$ from the hot flow are calculated using only the section of the time series between $t=0 s$ (the start of the measurement period) and $t=t_{\text {flash }}$ (the approximate flashback point) because the present study is concerned
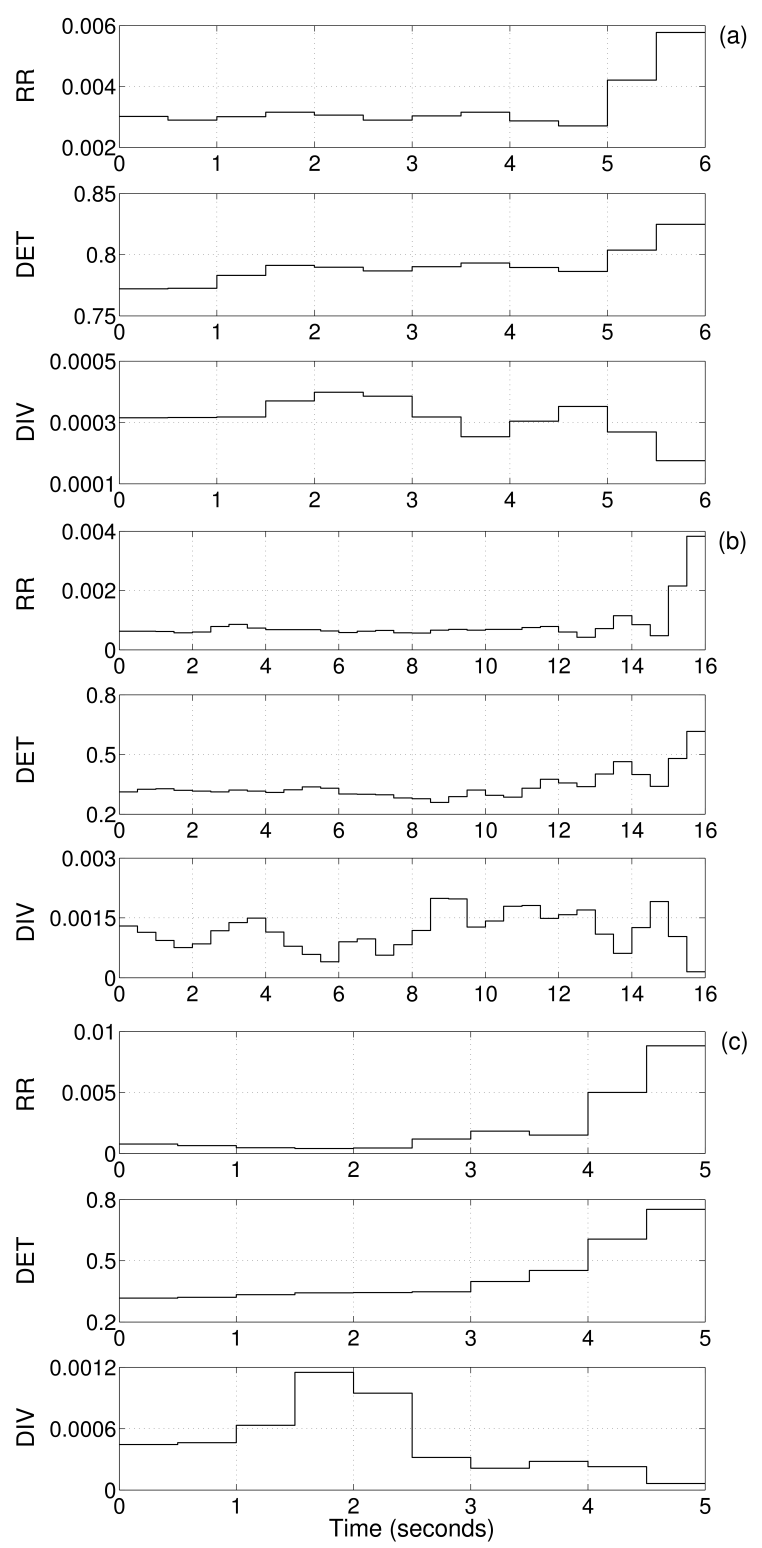

FIG. 9. The three RQA measures, recurrence rate (RR), determinism (DET) and divergence (DIV) calculated for the pressure time series (a) $P_{10}$, (b) $P_{12}$, and (c) $P_{14}$ using a one second long, running window with $50 \%$ overlap. The RQA measures are calculated for each pressure time series from $t=0 s$ to $t=t_{\text {flash }}$.

with the dynamic behaviour of the system prior to the flashback event.

The DET, which ranges from $0-1$, in Fig. 8 calculated for the pressure time series from the cold flow is very low and hence, indicates a highly stochastic process. This is expected in accordance with previous discussions on the isothermal flow. Although the DET is fluctuating slightly from window to window, the changes appear to be random: there are no significant trends present in the system dynamics.

The RR in Fig. 8 calculated for the pressure time series 
from the cold flow is low and does not change considerably over time. The magnitudes of $\mathrm{RR}$ correspond to the choice of the threshold, $\epsilon=4-5 \% d_{A}$. Because the isothermal states are dynamically similar, RR curves for the different cases coincide with each other.

The DIV curves stay close to 0.5 for the isothermal cases. $\mathrm{DIV}=0.5$ corresponds to the prescribed threshold for the minimum line length: $l_{\min }=2$. The DIV calculations show that the isothermal cases are dynamically similar and the RPs are comprised primarily of isolated points.

The DET in Fig. 9 calculated for the pressure time series from the reacting flow is high $(\sim 0.85)$. This is in accordance with the visual interpretation of the RPs in Fig. 6 and Fig. 7, which consist of distinctly visible diagonal lines. The reacting flow cases are clearly more deterministic when compared to the isothermal flow due to the existence of thermoacoustic coupling. More importantly, the DET curves for $P_{10}, P_{12}$, and $P_{14}$ show a distinct trend prior to flashback: the values begin to increase towards the onset of flashback (the time axes in Fig. 9 begin at $t=0 s$ and end at the approximate flashback point $\left.t=t_{\text {flash }}\right)$.

A similar trend as found for the DET curves is also found for RR. The curves gradually increase until flashback occurs. It can be noted that the increase starts 2-4 seconds prior to $t_{\text {flash }}$.

Changes in the DIV curves are more difficult to generalize for the three cases from the reactive flow. For $P_{10}$, a jump occurs at $t_{\text {flash }}$. On the other hand, for $P_{12}$, the fluctuations in DIV increase gradually as flashback is approached; similar to the RR and DET curves for the case, the trend appears 4 seconds before flashback. For $P_{14}$, inferences regarding any trend in the DIV curve could not be obtained.

\section{DISCUSSION}

For a thermoacoustically unstable system undergoing flashback, the results reported indicate that subtle temporal changes occur in the dynamics of the coupling prior to flashback. These changes are made apparent through the characterization of the recurrences of phase space trajectories reconstructed from pressure time traces, in a multi-dimensional phase space.

The indication of precursor behavior is first seen in the RPs where, the patterns in the RP change as flashback is approached. A change in the distribution of deterministic structures (lines) and non-deterministic structures (isolated points) leads to a variation in the pattern of the recurrence plots. It is further seen, through RQA, that before flashback, the determinism in the system shows an increasing trend, as an increasing number of isolated black dots (isolated recurrences) come together to form diagonal lines (consecutive recurrences). The recurrence rate also increases simultaneously; this could be related to the fact that before flashback, there is a relative in- crease in the sections of trajectories recurring and hence, in the overall number of recurrences at the same $\epsilon$. In the transition from an asymptotic state before flashback to another attractor after flashback, while the actual flashback event might be an instantaneous process, changes that occur in system dynamics are not. The fact that the determinism also increases in the case of a thermoacoustically stable combustion system prior to flashback has also been identified in a recent study ${ }^{34}$ by the authors using the translation error method ${ }^{35}$.

These trends that have been identified point to the possibility that as flashback is approached, the dynamics of the flame-hence, also the flame-acoustic coupling - changes. The duration of relevant changes that occur prior to flashback span 2-4 seconds. There observations lead to two important implications: a) precursors to flashback exist, and with appropriate methods, it is possible to design flashback detection and control; and b) the phenomenon of flashback is a result of interactions that span a duration of seconds. In previous studies on the mechanisms of flashback ${ }^{6,8,13}$, discussions focus on the stages involved in the evolution of the flame and hydrodynamic structures during a flashback event. It is very likely that observable qualitative changes that have been associated with the occurrence of flashback are also related to changes in the recurrence measures obtained from the pressure time trace.

In this study, thermoacoustically induced flashback is investigated. It is expected that investigation of other types of flashback on the lines of the present investigation should lead to similar results on flashback precursors.

Recurrence analysis, which enables quantification of system complexity and determinism, appears to be an appropriate method to identify transitional changes/precursor behavior present in the very complex dynamics that are involved in the process of flame flashback.

\section{CONCLUSIONS}

In the process of flashback in a combustor, it is shown here that subtle changes occur in system dynamics seconds before the actual flashback event. While linear time- and frequency-domain analyses fail to identify these changes, a trend in dynamical characteristics is observed through recurrence plots and subsequent recurrence quantification. A gradual increase in the contribution from deterministic dynamics in the system prior to flashback was observed through recurrence analysis. These findings have important academic as well as industrial implications. From an academic point of view, the results show that relevant dynamical changes occur in the flame seconds before flashback. Future investigations on flashback mechanisms could benefit from this information. At the same time, the results also demonstrate the feasibility of advance detection approaches. 


\section{ACKNOWLEDGMENTS}

LC would like to thank the Engineering and Physical Sciences Research Council for funding his doctorate, the Chair of Fluid Dynamics at the Technische Universität Berlin for hosting his research stay, and the School of Engineering at the University of Glasgow for partly funding his stay. LK is grateful for the financial support from the Deutsche Forschungsgemeinschaft through project KA 3968/1-1.

${ }^{1}$ A. H. Lefebvre and D. R. Ballal, Gas Turbine Combustion: Alternative Fuels and Emissions (CRC Press, 2010).

${ }^{2}$ A. C. Benim and K. J. Syed, Flashback Mechanisms in Lean Premixed Gas Turbine Combustion (Academic Press, 2014).

${ }^{3}$ T. Lieuwen, Unsteady combustor physics (Cambridge University Press, 2012).

${ }^{4}$ A. Berlad and A. Potter, "Relation of boundary velocity gradient for flash-back to burning velocity and quenching distance," Combustion and Flame 1, 127-128 (1957).

${ }^{5}$ C. Eichler, G. Baumgartner, and T. Sattelmayer, "Experimental investigation of turbulent boundary layer flashback limits for premixed hydrogen-air flames confined in ducts," in Volume 2: Combustion, Fuels and Emissions, Parts $A$ and $B$ (ASME International, 2011); C. Eichler and T. Sattelmayer, "Premixed flame flashback in wall boundary layers studied by long-distance micro-PIV," Exp Fluids 52, 347-360 (2011).

${ }^{6}$ Y. Sommerer, D. Galley, T. Poinsot, S. Ducruix, F. Lacas, and D. Veynante, "Large eddy simulation and experimental study of flashback and blow-off in a lean partially premixed swirled burner," J. of Turbulence 5 (2004), 10.1088/1468-5248/5/1/037. ${ }^{7}$ N. Karimi, C. Heeger, L. Christodoulou, and A. Dreizler, "Experimental and theoretical investigation of the flashback of a swirling, bluff-body stabilised, premixed flame," Zeitschrift für Physikalische Chemie 229, 663-689 (2015).

${ }^{8} \mathrm{M}$. Konle and T. Sattelmayer, "Interaction of heat release and vortex breakdown during flame flashback driven by combustion induced vortex breakdown," Exp Fluids 47, 627-635 (2009); "Time scale model for the prediction of the onset of flame flashback driven by combustion induced vortex breakdown," J. Eng. Gas Turbines Power 132, 041503 (2010).

${ }^{9}$ L. Vaneveld, K. Hom, and A. K. Oppenheim, "Secondary effects in combustion instabilities leading to flashback," AIAA Journal 22, 81-82 (1984).

${ }^{10}$ J. . Keller, L. Vaneveld, D. Korschelt, G. Hubbard, A. Ghoniem, J. Daily, and A. Oppenheim, "Mechanism of instabilities in turbulent combustion leading to flashback," AIAA Journal 20, 254262 (1982).

${ }^{11}$ O. Tuncer, S. Acharya, and J. Uhm, "Dynamics, nox and flashback characteristics of confined premixed hydrogen-enriched methane flames," International Journal of Hydrogen Energy 34, 496-506 (2009).

${ }^{12}$ C. J. Marek, L. C. Papathakos, and P. W. Verbulecz, "Preliminary studies of autoignition and flashback in a premixingprevaporizing flame tube using jet-a fuel at lean equivalence ratios," Tech. Rep. NASA-TM-X-3526 (NASA Lewis Research Center, Cleveland, OH, United States, 1977).

${ }^{13}$ C. Heeger, R. L. Gordon, M. J. Tummers, T. Sattelmayer, and A. Dreizler, "Experimental analysis of flashback in lean premixed swirling flames: upstream flame propagation," Exp Fluids 49, 853-863 (2010)

${ }^{14}$ M. Konle, F. Kiesewetter, and T. Sattelmayer, "Simultaneous high repetition rate PIV-LIF-measurements of CIVB driven flashback," Exp Fluids 44, 529-538 (2007).
${ }^{15}$ L. Kabiraj and R. I. Sujith, "Nonlinear self-excited thermoacoustic oscillations: intermittency and flame blowout," J. Fluid Mech. 713, 376-397 (2012).

${ }^{16}$ H. Gotoda, Y. Shinoda, M. Kobayashi, Y. Okuno, and S. Tachibana, "Detection and control of combustion instability based on the concept of dynamical system theory," Physical Review E 89 (2014), 10.1103/physreve.89.022910.

${ }^{17} \mathrm{H}$. Poincaré, "Sur la probleme des trois corps et les équations de la dynamique," Acta Mathematica 13, 1-270 (1890).

${ }^{18}$ J.-P. Eckmann, S. Kamphorst, and D. Ruelle, "Recurrence plots of dynamical systems," Europhys. Lett. 5, 973-977 (1987).

${ }^{19}$ N. Marwan, M. C. Romano, M. Thiel, and J. Kurths, "Recurrence plots for the analysis of complex systems," Physics Reports 738, 237-329 (2007).

${ }^{20} \mathrm{H}$. Abarbanel, "The analysis of observed chaotic data in physical systems," Reviews of Modern Physics 65, 1331-1392 (1993).

${ }^{21}$ G. M. Mindlin and R. Gilmore, "Topological analysis and synthesis of chaotic time series," Physica D 58, 229-242 (1992).

${ }^{22}$ D. Eroglu, N. Marwan, S. Prasad, and J. Kurths, "Finding recurrence networks' threshold adaptively for a specific time series," Nonlinear Processes in Geophysics 21, 1085-1092 (2014).

${ }^{23}$ N. Marwan, J. F. Donges, Y. Zou, R. V. Donner, and J. Kurths, "Complex network approach for recurrence analysis of time series," Physics Letters A 373, 4246-4254 (2009).

${ }^{24}$ J. F. Donges, R. V. Donner, M. H. Trauth, N. Marwan, H. J. Schellnhuber, and J. Kurths, "Nonlinear detection of paleoclimate-variability transitions possibly related to human evolution," Proceedings of the National Academy of Sciences of the United States of America 108, 20422 (2011).

${ }^{25}$ J. P. Zbilut and C. L. J. Webber, "Embeddings and delays as derived from quantification of recurrence plots," Physics Letters A 171, 199-203 (1992).

${ }^{26}$ B. Babaei, R. Zarghami, H. Sedighikamal, R. SotudehGharebagh, and N. Mostoufi, "Selection of minimal length of line in recurrence quantification analysis," Physica A 395, 112120 (2014).

${ }^{27}$ R. Balachandran, B. Ayoola, C. Kaminski, A. Dowling, and E. Mastorakos, "Experimental investigation of the nonlinear response of turbulent premixed flames to imposed inlet velocity oscillations," Combustion and Flame 143, 37-55 (2005).

${ }^{28}$ L. Kabiraj, A. Saurabh, N. Karimi, A. Sailor, E. Mastorakos, and A. Dowiling, "Chaos in an imperfectly premixed model combustor," Chaos: An Interdisciplinary Journal of Nonlinear Science 25, 023101 (2015).

${ }^{29}$ A. M. Hurst, T. R. Olsen, S. Goodman, J. VanDeWeert, and T. Shang, "An experimental frequency response characterization of mems piezoresistive pressure transducers," Proceedings of ASME Turbo Expo 2014: Turbine Technical Conference and Exposition 6, GT2014-27159 (2014).

${ }^{30}$ H. Gotoda, H. Nikimoto, T. Miyano, and S. Tachibana, "Dynamic properties of combustion instability in a lean premixed gas-turbine combustor," Chaos: An Interdisciplinary Journal of Nonlinear Science 21, 013124 (2011).

${ }^{31}$ C. L. Webber and J. P. Zbilut, "Dynamical assessment of physiological systems and states using recurrence plot strategies," Journal of Applied Physiology 76, 965-973 (1994).

${ }^{32}$ J. P. Zbilut, J. M. Zaldivar-Comenges, and F. Strozzi, "Recurrence quantification based liapunov exponents for monitoring divergence in experimental data," Physics Letters A 297, 173-181 (2002).

${ }^{33}$ Y. Peng and Z. Sun, "Characterization of qt and rr interval series during acute myocardial ischemia by means of recurrence quantification analysis," Medical and Biological Engineering and Computing 49, 25-31 (2011).

${ }^{34}$ L. Christodoulou, L. Kabiraj, A. Saurabh, J. Weinkauff, A. Dreizler, and N. Karimi, "Detection of flame flashback precursor using the translation error method," 10th Asia-Pacific Conference on Combustion, Beijing, China, July 2015.

${ }^{35}$ R. Wayland, D. Bromley, D. Pickett, and A. Passamante, "Recognising determinism in a time series," Physical Review Letters 70, 580-582 (1993). 